\title{
ERRATUM
}

\section{Solving Horn Clauses on Inductive Data Types Without Induction - ERRATUM}

\section{EMANUELE DE ANGELIS, FABIO FIORAVANTI, ALBERTO PETTOROSSI, and MAURIZIO PROIETTI}

\begin{abstract}
doi:10.1017/S1471068418000157, Published by Cambridge University Press, 10 August 2018.
\end{abstract}

A few lines in the original article (De Angelis et al. 2018) were printed incorrectly.

On page 454 line 5 the definition of the type tree should read:

type tree $=$ Leaf | Node of int $*$ tree $*$ tree ;

On page 455 line 14 the definition of Types should read:

Types $\ni \tau::=$ int $\mid$ bool $\mid$ ident $\mid \tau_{1} * \ldots * \tau_{k}$

On page 455 lines 24-26 the definitions of Values and Terms should read:

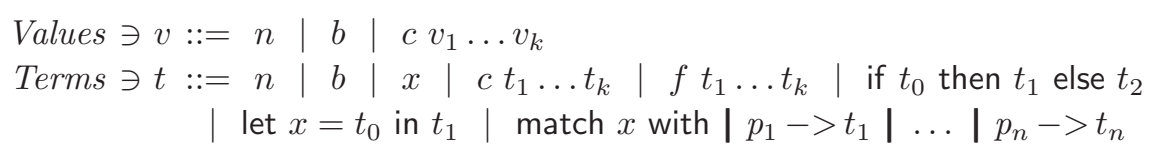

\section{Reference}

De Angelis, E., Fioravanti, F., Pettorossi, A., And Proietti, M. 2018. Solving Horn Clauses on Inductive Data Types Without Induction. Theory and Practice of Logic Programming 18, 3-4, 452-469. 